\title{
Examining the Structure of Lean and Agile Values Among Software Developers
}

\section{Fagerholm, Fabian}

Springer Science+Business Media

2014-05-26

Fagerholm , F \& Pagels, M E A 2014 , Examining the Structure of Lean and Agile Values Among Software Developers . in Agile Processes in Software Engineering and Extreme Programming : 15th International Conference, XP 2014, Rome, Italy, May 26-30, 2014. Proceedings . Springer Science+Business Media , pp. 218-233, International Conference on Agile Software Development (XP 2014) , Rome , Italy , 26/05/2014 . https://doi.org/10.1007/978-3-319-06862-6_15

http://hdl.handle.net/10138/136259

https://doi.org/10.1007/978-3-319-06862-6_15

acceptedVersion

Downloaded from Helda, University of Helsinki institutional repository.

This is an electronic reprint of the original article.

This reprint may differ from the original in pagination and typographic detail.

Please cite the original version. 


\title{
Examining the Structure of Lean and Agile Values Among Software Developers
}

\author{
Fabian Fagerholm and Max Pagels \\ Department of Computer Science, University of Helsinki \\ P.O. Box 68, FI-00014 University of Helsinki, Finland \\ fabian.fagerholm@helsinki.fi, max.pagels@cs.helsinki.fi
}

\begin{abstract}
Gaining maximum benefit of Lean and Agile methods requires a thorough understanding of their assumptions regarding culture, mindset, and values. This paper examines the value system structure of experienced developers working with Lean and Agile methods, and compares it to universal human values and individual personality. We developed and deployed an online survey on Lean and Agile values, with embedded measures for universal values and personality. The resulting data set, with 61 respondents, was analysed using agglomerative hierarchical clustering and multidimensional scaling. A value structure containing 11 Lean and Agile values was uncovered, yielding insight into how Lean and Agile developers experience values in their work. The analysis shows that Lean and Agile values are connected, but not equal, to universal values and personality. The proposed model can help practitioners understand the ethos of Lean and Agile methodologies and to assess their organisational culture. It may also help researchers to study models of software developer experience and value systems.
\end{abstract}

Key words: values, Agile software development, Lean software development, survey, quantitative study, developer experience, human factors

\section{Introduction}

In contemporary organisations, software teams often consist of people with varied cultural backgrounds and different personal characteristics. While diversity in knowledge and expertise can improve team performance, diversity in personal values can lead to conflict and lower performance [1]. Several studies indicate that diversity enhances performance by broadening the perspectives of work groups [2]. A shared professional culture can help individuals overcome differences in personal values and perform better. Culture and values have been considered important in software development for several reasons. In an essay on the history of the Agile Manifesto, Jim Highsmith, one of the founders of the Agile movement, notes [3]:

At the core, I believe Agile Methodologists are really about "mushy" stuff about delivering good products to customers by operating in an environment that does more than talk about "people as our most important asset" but 
actually "acts" as if people were the most important, and lose the word "asset". So in the final analysis, the meteoric rise of interest in - and sometimes tremendous criticism of - Agile Methodologies is about the mushy stuff of values and culture.

Integrating culture and value concerns into the software development process is a promising way to enhance developer experience [4]. While the importance of these issues is easy to understand, values themselves are very difficult to grasp. There is a lack of research illuminating the structure of values among software developers working with Lean and Agile methodologies. Many previous studies attempting to grasp the level of "agility" or "leanness" resort to checklists of methodological procedures. An improved understanding of the values that form the basis of individual reasoning and action, and their relationship to normative values in the Lean and Agile software development philosophies, would allow a discourse that goes beyond the practices that have been documented to date. In this paper, we report on a quantitative survey study which examines the structure of the Lean/Agile value system among professional software developers.

The rest of this paper is structured as follows. In Section 2, we first examine theories of human values; belief systems that influence evaluation of events and choice of action in broad and general terms. We then examine literature on Lean and Agile approaches in both software engineering and other fields, in order to gain a picture of possible value dimensions. In Section 3, we describe the research design: the research questions, survey design, sampling, survey deployment, and methods of analysis. In Section 4, we report the results of the study in detail. In Section 5, we discuss the findings and limitations of the study. Finally, in Section 6 , we summarise the findings and outline some possible future directions.

\section{Related Work}

Software engineers' motivation has received much systematic attention $[5,6,7]$. Motivation is moderated by complex socio-cognitive factors, including the cultural context with its value system and individual personal characteristics [6]. Values form an important component in motivation [8], and, thus influence performance and overall work experience. An understanding of values is therefore vital for improving many outcomes, including motivation and developer experience [4].

\subsection{Theories of Human Values}

Human values are deeply rooted, abstract motivations that guide, justify, or explain attitudes, norms, opinions, and actions [9]. Values constitute concepts or beliefs that serve as standards for desirable end-states or behaviours. They are deeply linked to affect and the self-concept [10]: when a value is activated in an individual, a corresponding feeling also occurs. Values prime attitudes and guide the selection of behaviours and events $[10,11,12,13]$. They are ordered by relative importance, forming a system of priorities to guide action [9]. Values are 
sometimes divided into terminal and instrumental values (e.g. [10]). Terminal values represent desirable end-states of existence - the goals that a person would like to achieve during their lifetime. Instrumental values represent preferable modes of behaviour - the means of achieving the terminal values. Values have practical consequences in many everyday situations. They are the most abstract type of social cognition used to guide general responses to classes of stimuli [14]. Values can guide the selection of behaviours and evaluation of events [13], and they impact organisational outcomes [15], business ethics [16, 17], and managerial behaviour [18]. It should be noted that values do not focus on specific objects or situations $[10,8]$. They explain behavioural patterns over longer periods of time rather than specific behaviours in particular situations [19].

Values are thought to stem from three universal requirements for human existence: individual biological needs; the preconditions for coordinated social action; and the survival and welfare needs of groups [13, 12]. Values are influenced by the cultures in which humans live. Hofstede's extensive work on values found four basic dimensions on which national cultures differ: Power Distance (the degree of inequality considered normal), Uncertainty Avoidance (preference for structured situations), Individualism (preference to act as an individual rather than as a group), and Masculinity (valuing things, power, and assertiveness more than people, quality of life, and nurturance) [20].

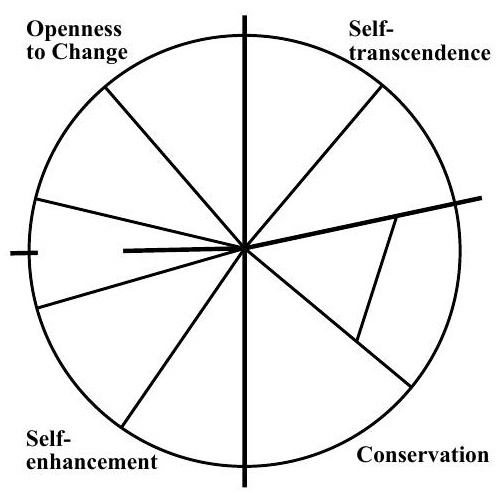

Fig. 1. Theoretical circumplex structure of relations among motivational types of values in the Schwartz model. (Adapted from Bilsky \& Schwartz [21].)

The Schwartz $[9,12,13]$ model of individual value differences has been widely used in social and cross-cultural psychology. The theory has been extensively verified in numerous countries, and postulates that values form a circumplex of related motivations, so that adjacent values in the model are mutually compatible (Figure 1). For example, the shared motivational emphasis in the combination stimulation - self-direction is an intrinsic motivation for mastery and openness to change, while the combination stimulation - hedonism represents a desire for affectively pleasant arousal [9]. 


\subsection{Values in Lean and Agile Software Development Methodologies}

Values play a special role in software development methodologies. Lean and Agile concepts have become commonplace in today's software development landscape. Both approaches have a core set of values that establish their fundamental philosophies, forming a base for more practical rules and methods.

Lean Values The term Lean Software Development was introduced in 2003 through the book "Lean Software Development: An Agile Toolkit" [22]. This treatment of Lean is positioned in relation to Agile software development. However, the roots of Lean thinking extends further back in history. Lean, or Toyota Production Systems (TPS), are just-in-time manufacturing systems initially developed at Toyota during 1948-1975 [23, 24]. This approach concerns not only manufacturing procedures, but is a comprehensive management philosophy - a foundation for competitive strength that relies on a deeply ingrained corporate culture [25]. In 2004, Jeffrey Liker published "The Toyota Way", introducing Toyota's corporate culture and the TPS to a large audience and popularising the Lean manufacturing philosophy [26].

The Toyota Way is the foundation of TPS [25, 26]. It is a summary of the managerial convictions and value systems inherited within Toyota as tacit knowledge; a code of conduct for its employees at all levels [25]. Numerous companies have attempted to emulate the structural parts of Lean, but have not succeeded in introducing the accompanying organisational culture and mindset [27]. Several authors have argued that Lean cannot be implemented effectively without also implementing the underlying value system [25, 27, 26, 28, 29, 23]. Liker attributes the difficulties in introducing Lean to a disregard for the Toyota Way [26, 25]. Liker points out that managerial understanding of the Toyota Way, and the ability to instil this thinking into the minds of workers, is essential in order to raise the level of lean companies [26].

Several authors have summarised essential Lean thinking. According to Womack and Jones [30] and Liker [26], Lean focuses on identifying customer value and delivering it by letting the product flow uninterrupted through a series of value-adding processes. Thus waste becomes visible and can be eliminated. Liker lists 14 Lean principles, including emphasising "a long-term philosophy, even at the expense of short-term financial goals"; levelling workload to work "like the tortoise, not the hare", building "a culture of stopping to fix problems, to get quality right the first time", "[growing] leaders who thoroughly understand the work, live the philosophy, and teach it to others", making decisions "slowly by consensus, [...] considering all options", and "[becoming] a learning organization through relentless reflection and continuous improvement" [26].

Agile Values In manufacturing, Agile is often considered the next step after Lean, but the two may also be viewed as complementary [31]. Agile software development was a response to perceived difficulties stemming from a turbulent business environment [32,33]. Though the term Agile software development was introduced in its namesake manifesto in 2001 [34], few of its ideas are new. Larman and Basili note that incremental and iterative development was 
in use as early as 1957 [35], making the approach several decades old. The initial driving force behind Agile software development consisted of practitioners and consultants [36]. Combined with a lack of rigorous conceptual studies, the result is a fragmented understanding of Agile, unclear definitions and even direct contradictions. Meanwhile, a unique characteristic of Agile software development is that it was founded on a set of core values [34]:

1. Individuals and interactions over processes and tools.

2. Working software over comprehensive documentation.

3. Customer collaboration over contract negotiation.

4. Responding to change over following a plan.

However, the perception and understanding of these values among practitioners is not necessarily literal. A critical analysis [37] examined the discourse of key methodological contributors using Lasswell's value framework [38], and found strong expressions of enlightenment - valuing knowledge and insight - and power valuing possibly coercive influence to affect policies. Wealth and skill were present to a lesser degree; rectitude, respect, affection, and well-being were only weakly expressed. The study claims that this is a legitimisation strategy to improve diffusion and industry adoption of Agile [37]. There is thus room to consider whether the true values of Lean and Agile practitioners are different. As practical enactments are often a combination of Lean and Agile, it is justified to examine the value structure of both methodologies as a single expanded set. While Lean and Agile can be considered complementary and partially overlapping, it is not clear how the combination of their underlying value systems are understood by practitioners, a gap that this study aims to address.

\section{Research Design and Execution}

We took a quantitative survey approach to examine the structure of Lean and Agile values among software developers. We sought to expand the understanding of this construct by addressing the following research questions:

RQ1 What is the structure of the Lean and Agile value system among software developers?

RQ2 What is the relationship between the Lean and Agile value system and the general human value system?

RQ3 What is the relationship between the Lean and Agile value system and individual personality?

\subsection{Survey Design}

We designed a survey with inventories on human values, personality, and Lean/Agile values. The survey was piloted twice with a small number of students to adjust length, improve item wording, and remove low-quality items. We used the Schwartz Portrait Values Questionnaire (PVQ) [12] to obtain a measure of human values for each respondent. The PVQ measures the ten basic value 
orientations shown in Figure 1. It consists of 21 short verbal descriptions, portraits, of different people, each implying an orientation towards a single value type. For each, respondents must answer the question "How much like you is this person?" on a six-point scale. We used the Ten-Item Personality Inventory (TIPI) [39], a short instrument for assessing personality according to the Big Five personality model. While being short, the TIPI correlates well with established instruments such as the BFI, NEO-FFI, and NEO-PI-R, and although it does not allow assessment of individual sub-scales, it is suitable for research purposes when personality is not the main topic of research [39]. To assess Lean/Agile values, we devised a set of value statement items. These were derived from multiple literature sources, including research articles and books aimed for practitioners. Respondents were asked to indicate their agreement with each statement on a seven-point Likert scale. We included several different wordings of items, and items that were not explicitly given by any single source, but were implied. The survey included a total of 94 items, available as supplementary material [40].

\subsection{Sample and Survey Deployment}

Our main focus at this stage of research was Finnish software developers who work with Lean and/or Agile software development methods. However, we also allowed respondents from other countries to participate. We deployed the survey online during February to June 2013, and recruited participants from several sources where we knew experienced software development professionals could be reached. We directly contacted three companies in Finland; each agreed to let one of their teams participate in the survey. In addition, we obtained a number of respondents through social media discussion forums on relevant topics. Finally, we ran a Google AdWords campaign, promoting the survey to people who searched for related topics. Respondents were also recruited at scientific conferences with industry participants.

\subsection{Analysis Methods}

We used agglomerative hierarchical clustering and nonmetric multidimensional scaling to examine the structure of the responses. Agglomerative hierarchical clustering is a statistical data analysis method [41]. In our case, each survey response can be characterised as a point in a value space where Lean/Agile value items constitute the spatial dimensions. The clustering initially considers each point as an individual cluster, working bottom-up to iteratively join them based on similarity, i.e. closeness in the multidimensional space. The resulting tree structure reveals (dis)similarities in the data. We used cluster averages for agglomeration, and correlation as the distance metric.

Multidimensional scaling (MDS) is a technique for reducing the dimensionality of a data set while retaining its discriminative properties [42]. MDS can

collapse a data set into a two-dimensional representation, allowing an accessible visual representation of the data for interpretation. While value structures have 
previously been examined using theory-based MDS, where value dimensions are assigned initial locations, we chose not to use such an approach. The reasons for this are twofold. First, our aim at this stage is not to confirm an articulated hypothesis on Lean/Agile value structures but to explore the construct. Second, current literature is too fragmented to support a single theory-based hypothesis. This is contrary to the situation in, e.g., research on general human values, where such theoretical support does exist (see e.g. [21]).

\section{Analysis and Results}

Of 61 received responses, 57 were retained after cleaning the data. Unfortunately, due to several avenues used to contact potential respondents, the response rate of the survey could not be determined. The respondents were between 22 and 62 years of age, with a median age of 35 years. 47 respondents were male, 7 female, and 3 did not disclose their gender. Respondents' country of birth was Finland (65\%), Germany (7\%), Sweden (7\%), Turkey (4\%) and USA (4\%); other countries (United Kingdom, Bangladesh, The Netherlands, Italy, Russia, Israel and China) formed the remaining $13 \% .72 \%$ of respondents currently lived in Finland, and $63 \%$ were of Finnish nationality. 14\% had completed high school or vocational education, $31 \%$ had a Bachelor's degree, and $46 \%$ a Master's degree. $86 \%$ were currently employed. The median years of work experience was 12 . Respondents reported working in a wide range of positions, including software development, testing, architecture, and coaching, product management, and consulting. A small number of company owners and top management members responded to the survey. Organisations ranged from small (less than 10 employees) to large (more than 1000 employees), with the mean size being between 100 and 499 employees. The demographics match the intended population, with a relevant background and level of professional experience.

\subsection{Structure of Lean and Agile Values}

Figure 2 shows the hierarchical clustering of the Lean/Agile values data. Ten clusters, highlighted with a solid surrounding box, have a confidence level of $p \geq 0.9$, indicating a large degree of support. An additional cluster and three expanded clusters, indicated by dashed lines, have somewhat weaker confidence levels ( $p \geq 0.8$ or higher) but are theoretically motivated. We examine the contents of these clusters from left to right.

Valuing a Narrow Work Focus The items in the first cluster represents the view that software developers should focus on their technical work and not deal with stakeholders or management of work: "Programmers are supposed to write code, and it's not their responsibility if tasks overlap or are unclear" (v80), "User needs might be important, but software developers should focus on the implementation details" (v22), "The main thing is just to get the work done, it's not my job to figure out work processes" (v65). This is contrary to ideas of 
Cluster dendrogram with AU values (\%)

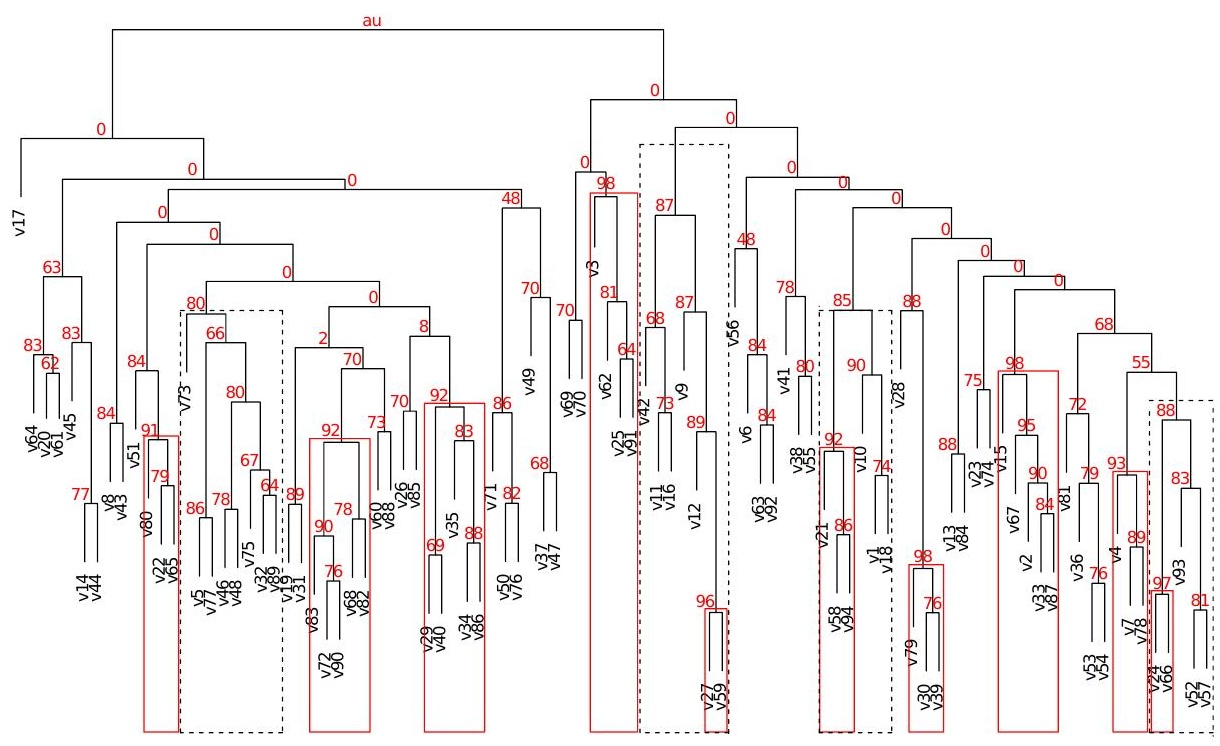

Fig. 2. Hierarchical clustering dendrogram of Lean/Agile values data. Variables are shown as leaves in the tree. The numbers in the branch junctions indicate the $\mathrm{p}$-value of the corresponding subtree. The value (AU) is the "approximately unbiased" p-value [43]. Clusters with $p \geq 0.9$ (AU) are marked with a solid box; theoretically motivated clusters are marked with a dashed-line box.

self-organisation, process ownership, continuous self-improvement, and inclusion of the customer in the development process - notions that are valued in Agile development.

Valuing Flexibility in Task Execution and Leadership The second cluster includes beliefs concerning flexibility. "I try to be flexible and if someone has an important task that is not in the iteration backlog, I do it anyway" (v73), "Working on many things simultaneously makes me more productive" (v5), and "I have no problem switching tasks even though I have already started another task" (v77) (task-level flexibility). "Several product owners is better than one product owner" (v46), and "To get more done in software projects, you must work longer hours" (v48) point to flexibility in work direction and amount. One person working on too many tasks simultaneously is against Agile and Lean principles; working longer hours and having several product owners can be seen as having an adverse effect on the ability to maintain a constant pace of development, something that both Agile and Lean philosophies strive to achieve.

"Asking someone who already knows the answer to a question is always better than figuring out the solution oneself" (v75), "The best software developers are highly specialised and focus on their speciality" (v32), and "Only those with the greatest knowledge and highest expertise in one particular area should make 
decisions that have to do with that area" (v89) represent information-seeking flexibility, and the view that expertise should lead to decision-making authority. Agreement with these statements represent valuing a strong distinction between leader and follower, and giving up individual control to follow given instructions. The level of confidence in this cluster is lower than that of the other clusters $(p \geq 0.8)$.

Valuing Planning and Preparation The third cluster concerns the notion that planning and preparation are important: "There has to be someone with authority who regularly reviews and approves a team's work before it can continue" (v83), "Great software is the result of a great plan which is carefully followed" (v72), "To succeed in a software project, you must stick to the plan" (v90), "It is beneficial to prepare stories or task descriptions months or weeks in advance" (v68), and "Work should not start before exact tasks and specifications are ready" (v82). This cluster refers to planning and preparing before work starts - the "big plan up front". This is in conflict with the Agile avoidance of long-term planning, preferring reactivity, and relying on feedback from development iterations rather than detailed specifications. On the other hand, a long-term perspective, the belief that a high-quality process will produce high-quality results, and the preference for slow and thorough decision-making, are present in Lean thinking.

Valuing Adherence to the Process The fourth cluster represents the belief that processes should be strictly followed: "I prefer having everyone follow a process rather than interacting with people to agree on what to do next" (v29), "Even if something is broken in the software under development, the focus has to be on what was planned, not on fixing everything" (v35), "I prefer large software development teams rather than small ones" (v34), and "Great software is the result of carefully applying a great software development process" (v86). This view may be connected to a preference for working in large organisations (v34), where the impact of individual deviation is negligible: "It's ok to make up a feature in order to justify the use of the latest technology" (v40).

Valuing Discipline The fifth cluster emphasises discipline: "A development process has to be followed strictly and with discipline" (v3), "Team members should be able to justify why they use certain tools" (v62), and "Having no development process leads to chaos and failed projects" (v25). However, the statement "Great software is the result of constant replanning when changes occur" (v91) is also within this cluster, indicating that discipline does not preclude responsiveness to change.

Valuing Reliance on People The sixth cluster concerns valuing people, represented directly in the Agile Manifesto: "Having the best people in the project is more important than having the best development tools" (v27), and "Having the best people in the project is more important than spending time on managing the process" (v59). However, by including some additional items in this branch (Figure $2 ; p \geq 0.87$ ), we can see a more complicated picture: "People are more important for success than following a development process" (v12), "If something 
is broken in the software under development, it should be fixed immediately" (v9), "If an ongoing task can be finished very soon, it should always be finished even if there is a more important task pending" (v42), "It is very important for team members to know the contents of the contract(s) made with the paying customer" (v11), and "It is impossible to fully plan a software project" (v16). In this extended cluster, "valuing people" relates to responsiveness and knowledge of contractual obligations, and to the belief that planning a software project is impossible. In other words, this value is instrumental, aiming to increase performance, not necessarily to improve well-being.

Valuing the Freedom to Organise The seventh cluster concerns selforganisation and responsiveness: "In software development projects, I prefer to just solve problems as they come rather than thinking far ahead" (v21), "The best architectures and designs are created when teams can organise themselves" (v58), and "Software development team members should be allowed to organise themselves in any way they see fit" (v94). Extending the cluster with three additional items $(p \geq 0.85)$ yields a more complete picture: "Physically moving around a lot lowers my productivity" (v10), "Software developers should be allowed to freely choose any tools they wish to use" (v1), and "Software development team members should have the authority to choose what they work on" (v18). This cluster seems to be in opposition to the fifth cluster. High agreement here could represent valuing freedom for developers to learn and organise their work.

Valuing a Sense of Purpose The eighth cluster represents the value of knowing the purpose of one's work and its role for an end goal: "Not knowing who the end user is during a project is a big problem" (v79), "When implementing a feature, it is critical to know who needs it and why" (v30), and "All team members must have a clear understanding of who the software is intended for" (v39).

Valuing Predictability and Justification The ninth cluster concerns a desire for uncertainty reduction. It is related to the desire to base action on evidence and observation rather than prescribed rules or unjustified orders. "Before implementing a feature, its value should be tested on end users" (v15), "Requirements can change, but it should not be permitted to change requirements during an iteration" (v67), "I want to spend time on identifying and eliminating unnecessary work in software development projects" (v2), "Tasks should always be doable in one iteration" (v33), and "I need to feel sure that the goals set for each development iteration are achievable" (v87). This cluster highlights a preference for specific, justified, and time- or scope-limited commitment.

Valuing Collaboration The tenth cluster shows another side of valuing people: the desire for close, collaborative work: "I wish pair programming would always be used in the projects I work in" (v4), "When faced with a large problem, everyone in a software development team should stop what they are doing and work together to solve it" (v7), and "It is best to meet in person instead of calling or emailing" (v78). This cluster separates the instrumental people values in cluster 
four from the benefits of collaborative work, putting emphasis on the value of working together.

Valuing Broad Stakeholder Involvement The eleventh and final cluster concerns customer involvement: "Great software is the result of the customer constantly monitoring the project" (v24), and "Great software is the result of close collaboration with the paying customer" (v66). In some ways, this is the counterpart to the first cluster. By cluster inclusion criteria $(p \geq 0.88)$, we can gain a broader understanding: "Everyone in a team should know what all the others are working on" (v93), "My highest priority is to satisfy the customer by continuously delivering valuable software" (v52), and "Working software is the only right measure of project progress" (v57). Together, these items indicate valuing customer involvement, with the customer and team co-creating the software.

\subsection{Relationship to Human Values}

In order to answer RQ2, we examined how the Lean/Agile value dimensions obtained through clustering relate to general human values. Figure 3 shows a two-dimensional MDS of both sets of value dimensions. Although there are some relationships between the two, our Lean/Agile value dimensions exhibit a structure that is different from the universal values. On the horizontal axis, human value dimensions are mostly centred, with Lean/Agile dimensions towards either end. The latter can thus be seen as polarised variants of the former. This axis represents a continuum ranging from an open, inclusive, and self-enhancing view to more authoritative, plan-based, and conforming values. This axis can be seen

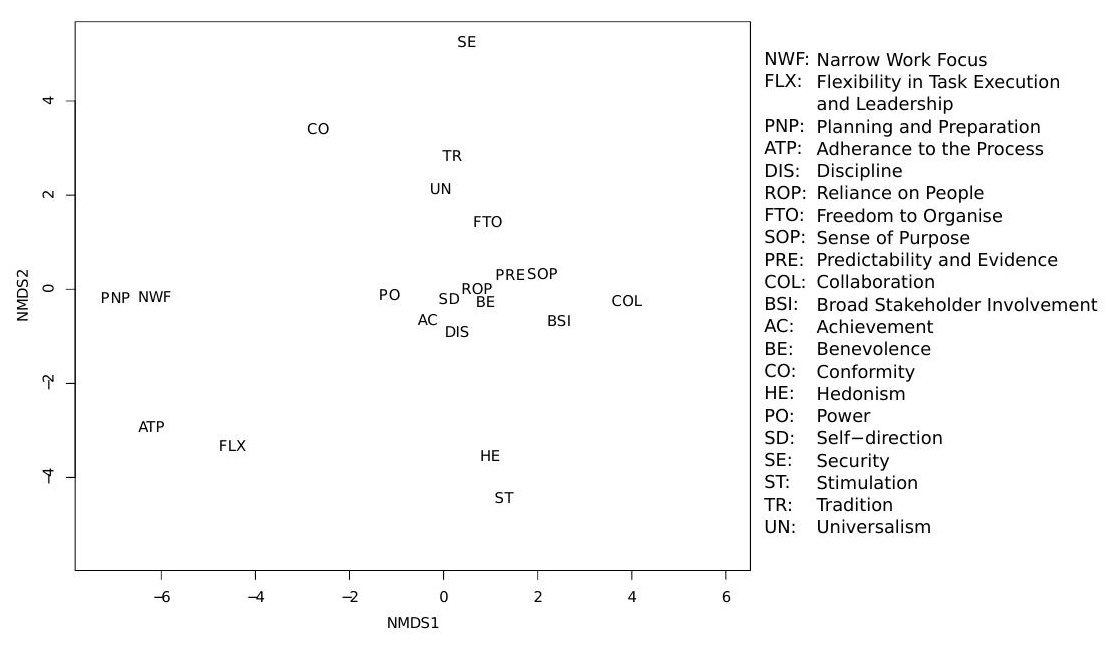

Fig. 3. Two-dimensional MDS of Lean/Agile value dimensions combined with Schwartz value data. The dimensions of the Lean/Agile values data are shown as variable names, and the Schwartz value dimensions are shown as two capital letters. 
as representing the traditional dichotomy between "bureaucratic" and "peopleoriented" views of software development approaches. The two value systems are more mixed on the vertical axis, but human values are present at either end. The dimensions in the lower end are related to openness to change, those in the middle are mixed, and conservation values are located in the high end. Lean/Agile values in the lower end can be interpreted as a relaxed attitude towards being led; the ones in the middle reflect a balanced and disciplined view required for collaborative work and group decision-making; the higher end reflects a self-oriented and more individualistic stance. This axis can be seen as a continuum of values regarding decision-making, control, and ambition that ranges from a focus on the self, through a collective view, and ends with carefreeness and flexibility to the degree of giving up control.

\subsection{Relationship to Personality}

Lean/Agile values may be expressions of individual personality. We therefore examine the relationship between Lean/Agile values and personality traits. Figure 4 shows an MDS of the Lean/Agile values with the Big Five personality traits. The data is scaled to a single dimension to gain an overall comparison. The scale can be considered as a continuum ranging from adherence to processes and roles, and submission to leadership, to a more collaborative and social approach to work. Extraversion, and to a slightly lesser extent, Agreeableness, tend towards the latter end of the continuum. Openness to experience is close to Reliance on People, but interestingly, Discipline also falls near this personality trait. The meaning of Discipline in the Lean/Agile values model may not refer to lack of imagination or creativity, but rather to a systematic approach to dealing with work. This corroborates the findings presented earlier (see Section 4.1, Discipline). Emotional Stability and Conscientiousness are closely located. However, they are far from the adherence end of the scale, indicating a stronger relation to the values of professional, systematic openness and creativity.

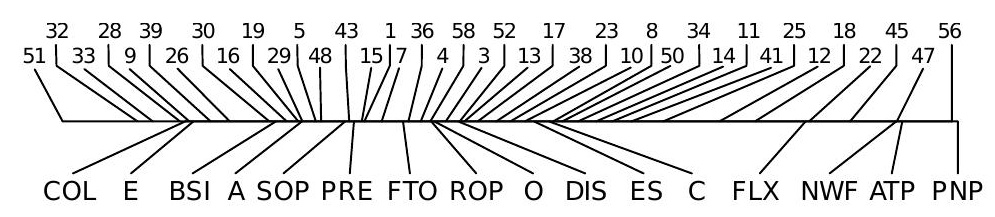

Fig. 4. One-dimensional MDS of Lean/Agile values combined with Big Five personality traits. The numbers represent each respondent, while the letters represent Lean/Agile values and personality traits. ES: Emotional stability, E: Extraversion, O: Openness to experience, A: Agreeableness, C: Conscientiousness. 


\section{Discussion and Limitations}

We are now in a position to answer our research questions. RQ1 concerned the structure of the Lean and Agile value system. Our analysis revealed eleven main dimensions (see Section 4.1), providing a model for the Lean and Agile value system. We can see that this model touches upon several aspects of software development work. The value structure reflects the holistic, practice-oriented approach in both Lean and Agile software development. It consists of a mixture of human aspects on individual and group levels, concerns regarding process adherence and flexibility, and notions of what is essential to meaningful work. The model is a foundation on which further studies can be built, as well a framework within which practitioners can position themselves.

RQ2 concerned the relationship between the value system and the general human value system. The largest differences lie on a continuum ranging from high preference for bureaucratic order to people-orientation, where differences in Lean/Agile values are more pronounced. Similarities exist on a continuum regarding type of decision-making, control, and ambition. Self-focus in universal values was congruent with valuing individual decision-making and self-enhancement; a collective focus was congruent with collaborative decision-making and benefiting the group; and a focus on personal pleasure was congruent with relinquishing personal ambition and following a direction chosen by others. Practitioners may want to consider their placement on this continuum and compare the value system of their local or corporate culture with their perception of Lean and Agile values.

RQ3 concerned the relationship between the Lean/Agile value system and individual personality. Our interpretation is that the two may be weakly linked. A preference for social values relates to the Extroversion/Agreeableness pair, while valuing the systematic, creative, and organisational side is more related to Openness to experience, Emotional stability, and Conscientiousness. However, values pertaining to adherence to processes, roles, and leadership, do not seem connected to personality. Valuing these dimensions may have more to do with company and national culture, schooling, and the business area in which a person works.

The largest threat to validity in this study is the limited sample. It is biased by lack of random selection and reliance on participant self-selection. It may not be representative of a larger Finnish population. However, based on the demographic characteristics of the sample, we argue that our results represent a reasonable starting point for empirically examining the Lean and Agile value construct. A second threat to validity is the possible bias in the survey instrument. We are aware that the instrument may lack some aspects of Lean and Agile thinking. However, the selection made here does represent a large number of aspects in the literature, and we argue that it provides reasonable coverage given the fragmented nature of the field. 


\section{Conclusions and Future Work}

In this paper, we reported on a study that investigated the value structure among Lean/Agile software developers. We found a model with eleven dimensions that structures Lean/Agile values as perceived by practitioners. Our analysis indicates that Lean/Agile values touch upon software development work as a whole rather than being limited to specific sub-areas. Comparing the value system to universal human values indicates that while there are some important links between the two, there are also areas where Lean/Agile values are more specific than universal human values. Some weak links between the Lean/Agile values and individual personality were also found.

The implications of our findings are twofold. First, practitioners can benefit from making implicit values more explicit in their work. Basing software development methodology on values can be beneficial: as values increase adaptive fitness by providing individuals with flexible patterns of behavioural response options [44], steering software development through values can be effective. In other words, rather than specifying actions for specific situations, a values-based approach allows practitioners to react dynamically in new and unforeseen situations. Also, a methodology that is compatible with cultural values has better odds of being accepted by practitioners, thus increasing chances for positive adoption. Being able to articulate the dimensions of the value system, rather than speaking in terms of methodological practices, could facilitate clarity and flexibility, increase opportunities for diversity in the workplace, and improve developer experience. This paper contributes a model which can be used for these purposes in practice.

Second, our findings have implications for future research. The construct validity of the model proposed in this paper should be tested further. With larger and more controlled samples, and by integrating the emerging literature on Lean software development, the combination of Lean and Agile, and the scaling of Lean and Agile methodologies, better validity may be obtained. Larger samples are also needed for other statistical techniques such as factor analysis. Apart from strengthening the results presented here, future research could benefit by examining Lean/Agile approaches from the perspective of culture and values rather than from traditional software engineering constructs such as methodologies or processes. As demonstrated in this paper, the often fuzzy and tacit understanding of Lean/Agile software development held by practitioners in the field can be made explicit by leveraging theory and research methods from social and behavioural sciences. In our own work, we aim to explore the possibilities of an improved sample to increase the breadth and validity of the results presented in this paper. We encourage other researchers to replicate our findings and expand the understanding of software development driven by values. 


\section{References}

1. Liang, T.P., Liu, C.C., Lin, T.M., Lin, B.: Effect of team diversity on software project performance. Industrial Management and Data Systems 107(5) (2007) 636-653

2. Patrick, H.A., Kumar, V.R.: Managing Workplace Diversity. SAGE Open (2012)

3. Highsmith, J.: History: The Agile Manifesto (2001) Accessed 2014-01-01.

4. Fagerholm, F., Münch, J.: Developer Experience: Concept and Definition. In: Proceedings of the International Conference on Software and System Process. (2012) 73-77

5. Beecham, S., Baddoo, N., Hall, T., Robinson, H., Sharp, H.: Motivation in Software Engineering: A systematic literature review. Information and Software Technology 50(9-10) (2008) 860-878

6. Sharp, H., Baddoo, N., Beecham, S., Hall, T., Robinson, H.: Models of motivation in software engineering. Information and Software Technology 51(1) (2009) 219-233

7. França, A., Gouveia, T., Santos, P., Santana, C., da Silva, F.: Motivation in software engineering: A systematic review update. In: 15th Annual Conference on Evaluation and Assessment in Software Engineering (EASE 2011). (2011) 154-163

8. Rokeach, M.: Understanding human values. Free press, New York (1979)

9. Schwartz, S.: Universals in the content and structure of values: Theoretical advances and empirical tests in 20 countries. Advances in experimental social psychology 25(1) (1992) 1-65

10. Rokeach, M.: The nature of human values. Free press, New York (1973)

11. Feather, N.T. In: Values, deservingness, and attitudes toward high achievers: Research on tall poppies. Lawrence Erlbaum Associates, Inc, Hillsdale, NJ (1996) $215-251$

12. Schwartz, S., Bilsky, W.: Toward a Theory of the Universal Content and Structure of Values: Extensions and Cross-Cultural Replications. Journal of Personality and Social Psychology 58(5) (1990) 878-891

13. Schwartz, S., Bilsky, W.: Toward A Universal Psychological Structure of Human Values. Journal of Personality and Social Psychology 53(3) (1987) 550-562

14. Kahle, L. In: Social values and consumer behavior: Research from the list of values. Lawrence Erlbaum Associates, Inc. (1996) 135-151

15. Weeks, W., Kahle, L.: Social values and salespeople's effort. Entrepreneurial versus routine selling. Journal of Business Research 20(2) (1990) 183-190

16. Feather, N.: Values, Valences, and Choice: The Influence of Values on the Perceived Attractiveness and Choice of Alternatives. Journal of Personality and Social Psychology 68(6) (1995) 1135-1151

17. Mumford, M., Helton, W., Decker, B., Connelly, M., Doorn, J.V.: Values and Beliefs Related to Ethical Decisions. Teaching Business Ethics 7(2) (2003) 139-170

18. Smith, P., Peterson, M., Schwartz, S.: Cultural Values, Sources of Guidance, and their Relevance to Managerial Behavior: A 47-Nation Study. Journal of CrossCultural Psychology 33(2) (2002) 188-208

19. Bond, M.H., Kwok, L., Schwartz, S.: Explaining Choices in Procedural and Distributive Justice Across Cultures. International Journal of Psychology 27(2) (1992) 211

20. Hofstede, G.: Culture's consequences: International differences in work-related values. Volume 5. Sage Publications, Inc. (1984)

21. Bilsky, W., Janik, M., Schwartz, S.: The Structural Organization of Human Values - Evidence from Three Rounds of the European Social Survey (ESS). Journal of Cross-Cultural Psychology 42(5) (2011) 759-776 
22. Poppendieck, M.: Lean Software Development: An Agile Toolkit. Addison-Wesley Professional (2003)

23. Ōno, T.: Toyota production system: beyond large-scale production. Productivity press (1988)

24. Ward, A.: Lean product and process development. Lean Enterprise Institute (2007)

25. Saruta, M.: Toyota Production Systems: The 'Toyota Way' and Labour-Management Relations. Asian Business \& Management 5(4) (12 2006) 487

26. Liker, J.: The Toyota Way: 14 Management Principles from the World's Greatest Manufacturer. McGraw-Hill, New York (2004)

27. Hines, P., Holwe, M., Rich, N.: Learning to evolve: A review of contemporary lean thinking. International Journal of Operations \& Production Management 24(9) (2004) 994-1011

28. Holweg, M., Pil, F.: Successful Build-To-Order Strategies Start With the Customer. MIT Sloan Management Review 43(1) (2001) 74-83

29. Womack, J.P., Jones, D., Roos, D.: The Machine That Changed the World. Simon \& Schuster (2007)

30. Womack, J.P., Jones, D.: Banish waste and create wealth in your corporation. Free press, New York (2003)

31. Naylor, J.B., Naim, M.M., Berry, D.: Leagility: Integrating the lean and agile manufacturing paradigms in the total supply chain. International Journal of Production Economics 62(1-2) (1999) 107-118

32. Cockburn, A., Highsmith, J.: Agile software development, the people factor. Computer 34(11) (2001) 131-133

33. Highsmith, J., Cockburn, A.: Agile software development: the business of innovation. Computer 34(9) (2001) 120-127

34. Alliance, T.A.: The Agile Manifesto (2001) Online: http://www.agilemanifesto.org/. Accessed 2014-01-01.

35. Larman, C., Basili, V.: Iterative and Incremental Development: A Brief History. Computer 36(6) (2003) 47-56

36. Conboy, K.: Agility from first principles: Reconstructing the concept of agility in information systems development. Information Systems Research 20(3) (2009) 329-354

37. Lawrence, C., Rodriguez, P.: The Interpretation and Legitimization of Values in Agile's Organizing Vision. Proceedings of the European Conference on Information Systems (ECIS) (2012) 10-13

38. Lasswell, H., Kaplan, A.: Power \& Society: a Framework for Political Inquiry. Yale University Press, New Haven (1950)

39. Gosling, S., Rentfrow, P., Swann, J.W.: A very brief measure of the Big-Five personality domains. Journal of Research in Personality 37(6) (2003) 504-528

40. Fagerholm, F.: Lean and Agile Values Survey 2013. Technical report (2014) Online: http://www.cs.helsinki.fi/people/fabian.fagerholm/agilevalues2013/. Retrieved 2014-01-04.

41. Johnson, S.: Hierarchical clustering schemes. Psychometrika 32(3) (1967) 241-254

42. Davison, M.: Introduction to Multidimensional Scaling and Its Applications. Applied Psychological Measurement 7(4) (1983) 373-379

43. Suzuki, R., Shimodaira, H.: pvclust: Hierarchical Clustering with P-Values via Multiscale Bootstrap Resampling. (2011) R package version 1.2-2.

44. Michod, R.E. In: Biology and the origin of values. Aldine de Gruyter, Hawthorne, NY (1993) 261-272 